\title{
Synthesis and characteristics of iron nanoparticles in a carbon matrix along with the catalytic graphitization of amorphous carbon
}

\author{
E.P.Sajitha ${ }^{\text {a,* }}$, V.Prasad ${ }^{\text {a }}$, S.V.Subramanyam ${ }^{\text {a }}$, S.Eto ${ }^{b}$, \\ Kazuyuki Takai ${ }^{\mathrm{b}}$, and T. Enoki ${ }^{\mathrm{b}}$ \\ ${ }^{a}$ Department of Physics, Indian Institute of Science, Bangalore, India \\ ${ }^{\mathrm{b}}$ Department of Chemistry, Tokyo Institute of Technology, Tokyo, Japan
}

\begin{abstract}
Iron nanoparticles in a carbon matrix were synthesized by in-situ pyrolysis of maleic anhydride and ferrocene, using different molecular weight percentages. The characterization and magnetic properties of the carbon-iron system were investigated systematically. Transmission electron microscope (TEM) images showed that the as-prepared samples consist of nanometric dark grains (iron-rich phase) embedded in a light matrix (carbon-rich phase). X-ray diffraction and TEM selected area diffraction revealed catalytic graphitization and iron phases present in the sample. The carbon-metal system shows a finite hysteresis loop even at room temperature indicating its ferromagnetic nature. The saturation magnetization equals the bulk iron carbide value at low temperature. The coercive force exhibits $1 / d$ dependance at low temperature having a maximum $\mathrm{H}_{C}$ of $2 \mathrm{kOe}$ for the lowest iron concentration sample.
\end{abstract}

Key words: A. Pyrolytic carbon; B. Chemical vapour deposition; C. X-ray diffraction, Transmission electron microscopy; D. Magnetic properties PACS: 82.30.Lp, 81.15.Gh, 61.10.Nz, 68.37.Lp, 36.40.Cg

\footnotetext{
* corresponding author Tel.: 191-80-2293-2313; fax: 191-80-2360-2602.

Email address: sajitha@physics.iisc.ernet.in (E.P.Sajitha).
} 


\section{Introduction}

Nanoparticles possessing magnetic properties embedded in a non-magnetic matrix have gained interest due to the properties they exhibit, like superparamagnetism [1], giant coercivity [2-4] etc. A non-magnetic matrix like carbon $[1,5-10], \mathrm{SiO}_{2}[2-4]$ etc. improves the magnetic stability in these nanosized particles by reducing the random flipping of the magnetic moment occurring by thermal fluctuations. Interest in carbon-metal systems is due to the possibility of obtaining nanocrystalline metal particles encapsulated by crystalline or amorphous carbon. Carbon coated ferromagnetic nanocrystallites have important applications where ferromagnetic iron oxide are currently being used such as magnetic data storage, magnetic toner in xerography, ferrofluid, biomedical applications etc $[11,12]$.

Several techniques have been developed for synthesizing carbon-coated metal nanocrystallites, such as the standard carbon arc technique [1,11], the modified tungsten arc technique [13], magnetron and ion-beam cosputtering [14], chemical vapour deposition $[15,16]$ and laser pyrolysis [17]. Chemical vapour deposition(CVD) of carbon from organic precursors, assisted by the catalytic activity of small transition metal particles has been the method for the production of carbon nanotubes $[16,18]$. The yield and nature of nanotubes depends on the preparation method and precursor used. This method has also gained interest in the synthesis of carbon nanocoils [19], nanospheres [16,18] and encapsulated metal particles $[15,16]$. Nanoparticles of iron in a carbon matrix, with enhancement in graphitization, are produced by ferrocene catalyzed pyrolysis of maleic anhydride $\left(\mathrm{C}_{4} \mathrm{H}_{2} \mathrm{O}_{3}\right)$ by a thermal CVD method. This method is also used to investigate the production of nanotubes using the precursor maleic anhydride with ferrocene acting as the catalyst source. Here we report the morphology, structure and the magnetic properties exhibited by the $\mathrm{C}: \mathrm{Fe}$ system.

\section{Experiment}

Nanocrystallites of iron embedded in an amorphous carbon matrix are prepared by in-situ pyrolysis of maleic anhydride $\left(\mathrm{C}_{4} \mathrm{H}_{2} \mathrm{O}_{3}\right)$ and ferrocene $\left(\mathrm{C}_{5} \mathrm{H}_{5}\right)_{2} \mathrm{Fe}$. Thermally assisted CVD is used to decompose and chemically react the introduced precursors. This method provides relative size control of the individual particles by varying $\mathrm{C} / \mathrm{Fe}$ concentration in precursors and the pyrolysis temperature during the co-decomposition process.

The experiment was carried out in a pre-evacuated $50 \mathrm{~cm}$ length, $10 \mathrm{~mm}$ diameter quartz tube with one end closed, and the other connected to an external 
bladder to collect the exhaust gases produced during pyrolysis. The furnace was heated at a rate of $8^{\circ} \mathrm{C} /$ min taking about 2 hours to reach the set temperature of $900^{\circ} \mathrm{C}$. The weight of maleic anhydride for pyrolysis was kept constant ( $196 \mathrm{mg})$ for all compositions. A change in iron content was made by changing the mass percent of ferrocene. The initial molecular weight ratios of maleic anhydride to ferrocene were 20:1, 10:1, 5:1, and 2:1 (i.e. $5 \%, 10 \%, 20 \%$ and $50 \%$ of ferrocene molecular weight). The samples are respectively designated C:Fe05, C:Fe10, C:Fe20, C:Fe50. The two precursors were placed at the closed end of the quartz tube, evacuated to $10^{-3}$ torr, flushed with argon gas and allowed to pyrolyse at $900^{\circ} \mathrm{C}$.

After one hour at the set temperature, the exhaust gases collected in the bladder were carefully removed in an argon atmosphere and replaced. The experiment was carried out at ambient pressure and the entire preparation process lasted about 5 hours, after which power was switched off and the furnace allowed to cool to room temperature. The powdery C:Fe samples were deposited mainly at the colder end of the furnace, and were collected by slightly scratching the surfaces of the quartz tube. A maximum yield of $3 \%$ was obtained depending on the amount of precursor used.

The structure and phases of the C:Fe samples were identified by x-ray diffraction with $\mathrm{CuK} \alpha$ radiation. The morphology of the particles was observed using transmission electron microscopy. SQUID magnetometer were used to measure magnetic properties of the samples in a magnetic field of $-55 \mathrm{kOe}$ to $+55 \mathrm{kOe}$.

\section{Results and Discussion}

\subsection{Structural Properties}

Powder x-ray diffraction (fig.1) of the as prepared powder sample shows the graphite (002) peak over the amorphous carbon background and the different phases of iron. The crystalline phases in the C:Fe composites were identified using Joint Committee for Powder Diffraction Standard cards. The (002) peak intensity increases as the iron percentage increases, along with the appearance of different phase of iron. Fig. 1 shows the iron carbide phase for a lower concentration of iron, whereas $\mathrm{Fe}_{3} \mathrm{O}_{4}$ and $\alpha$-Fe peaks starts appearing for higher concentration. The Scherrer formula is used to calculate the crystallite size $\mathrm{L}_{C}$ from the $\mathrm{G}(002)$ peak and values increase from $1.7 \mathrm{~nm}$ for amorphous carbon to 40nm for the C:Fe50 sample. The different phases of iron appearing with the increase in percent ferrocene are given in table 1. 


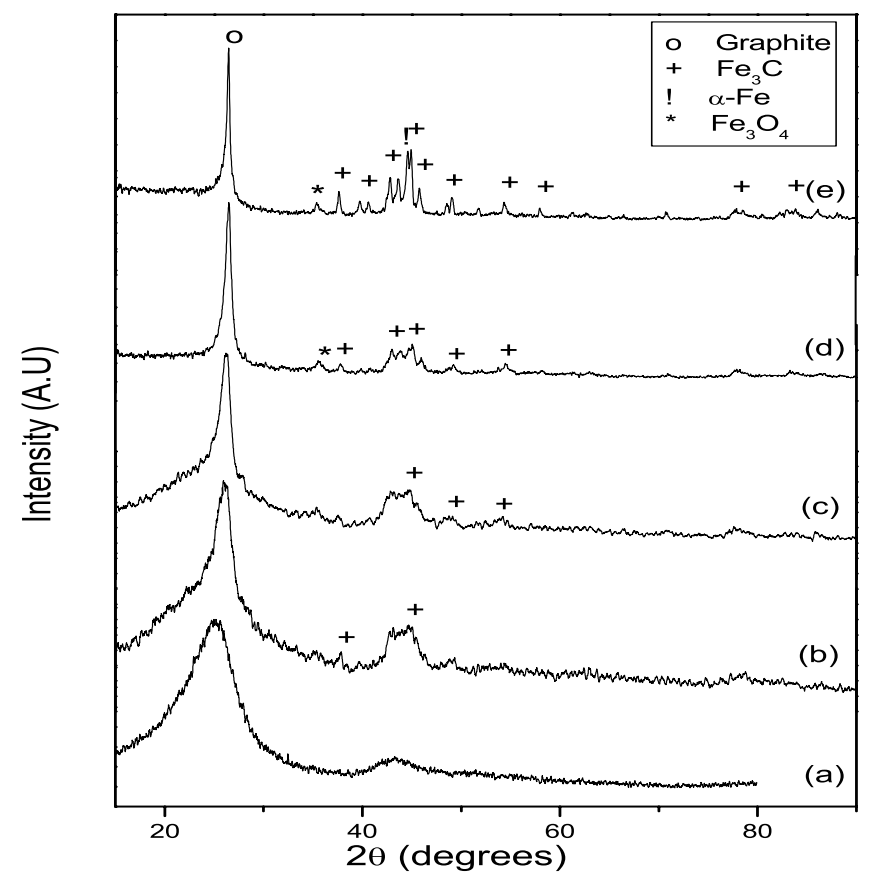

Fig. 1. X-Ray powder diffraction of C:Fe compositions (a)amorphous-C (b)C:Fe05 (c)C:Fe10 (d)C:Fe20 (e)C:Fe50 prepared at $900^{\circ} \mathrm{C}$ showing graphite (o), $\mathrm{Fe}_{3} \mathrm{C}(+)$, $\mathrm{Fe}_{3} \mathrm{O}_{4}(*)$ and $\alpha$-Fe (bcc) (!) peaks

Table 1

Different phases of iron in the C:Fe sample compositions

\begin{tabular}{|c|c|c|c|c|}
\hline Compositions & $\mathrm{C}: \mathrm{Fe} 05$ & $\mathrm{C}: \mathrm{Fe} 10$ & $\mathrm{C}: \mathrm{Fe} 20$ & $\mathrm{C}: \mathrm{Fe} 50$ \\
\hline Iron Phases & $\mathrm{Fe}_{3} \mathrm{C}$ & $\mathrm{Fe}_{3} \mathrm{C}$ & $\mathrm{Fe}_{3} \mathrm{C}, \mathrm{Fe}_{3} \mathrm{O}_{4}$ & $\mathrm{Fe}_{3} \mathrm{C}, \mathrm{Fe}_{3} \mathrm{O}_{4}, \alpha-\mathrm{Fe}$ \\
\hline
\end{tabular}

The graphitization of amorphous carbon by transition metal catalyst [20] is well documented in the literature. It involves the formation of an intermediate compound of metal carbide, which then recystallizes as metal crystals and graphite. If the process of graphitization is stopped before completion, we have a partially graphitized carbon matrix with iron carbide crystals embedded in it. The form and features of carbon particles obtained depend on the preparation conditions.

\subsection{Determination of Iron content}

The iron contents of the C:Fe05 and C:Fe50 samples were determined by heat treatment in open air at high temperature [7]. The residue obtained is of iron oxide mainly $\mathrm{Fe}_{2} \mathrm{O}_{3}$ as evidenced by X-Ray diffraction study (figure not shown). The weight of iron oxide is used to calculate the iron and carbon 

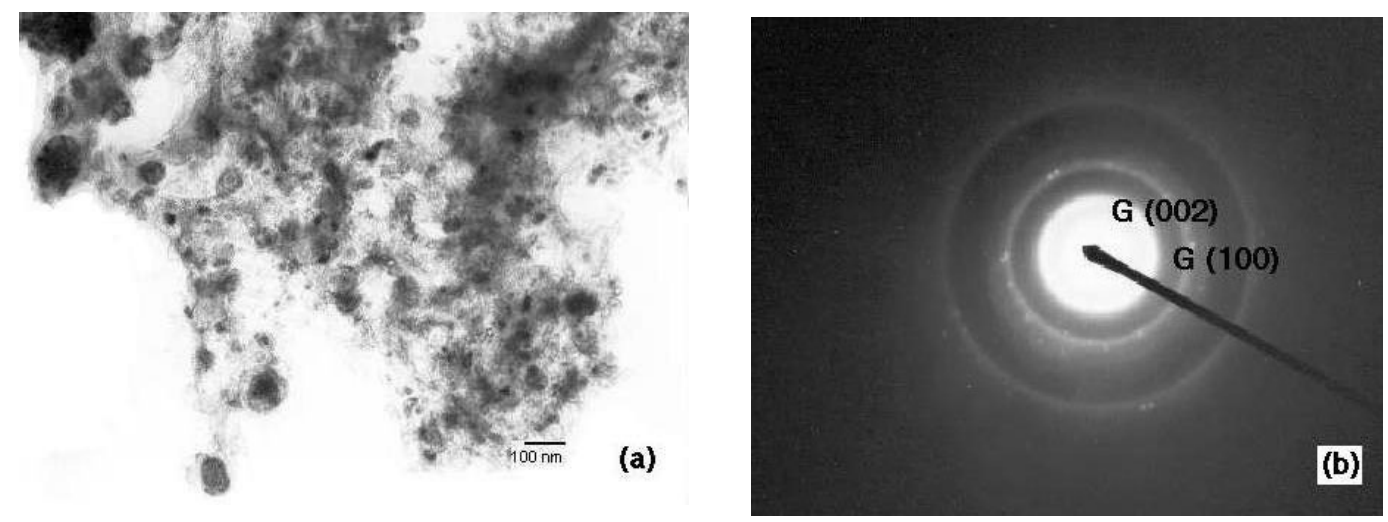

Fig. 2. (a)TEM image and (b) SAED of CFe05 sample
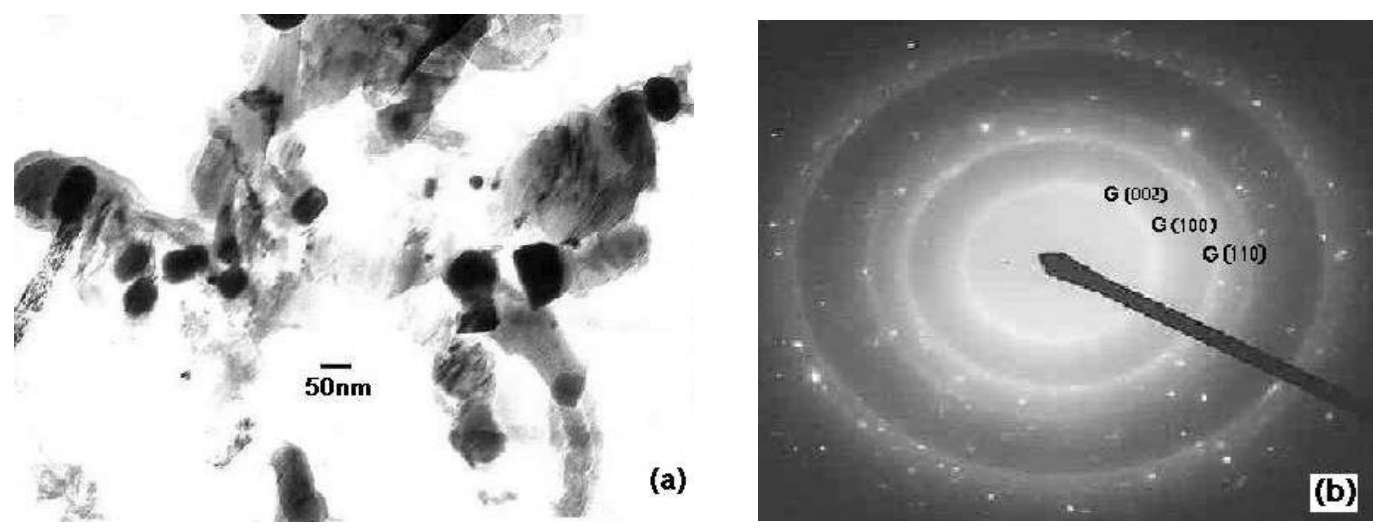

Fig. 3. (a)TEM image and (b) SAED of CFe50 sample

percentages in the sample. Initially the C:Fe05 and C:Fe50 samples weighed $0.070 \mathrm{~g}$ and $0.063 \mathrm{~g}$ respectively, while their oxidized residues weighed $0.009 \mathrm{~g}$ and $0.016 \mathrm{~g}$. Thus the iron weight percentage in the C:Fe05 sample is calculated to be $9 \%$ and that in the C:Fe50 sample to be $17.8 \%$, the balance being carbon.

\subsection{Morphology of the C:Fe samples}

As-prepared samples were dispersed in ethanol by ultrasonication and then put on copper grids for TEM observation. Figure 2 and 3 show typical TEM images of the as-deposited powder sample and the corresponding selected area electron diffraction (SAED) pattern. Figure 2 shows that the Fe nanocrystallites are well embedded in the carbon matrix. The presence of nanometric dark grains (iron-rich phase) embedded in a light matrix (carbon-rich phase) shows the granular morphology of the sample. The TEM image shows that the percentage of nanotubes in the compositions is negligible. All the diffraction rings are very broad and correspond to the $\mathrm{C}$ (002) plane and to nanometric $\mathrm{Fe}_{3} \mathrm{C}$ grains. In figure 3, larger iron clusters together with graphite flakes are seen. In the SAED pattern, sharp diffraction spots are present in addition to 


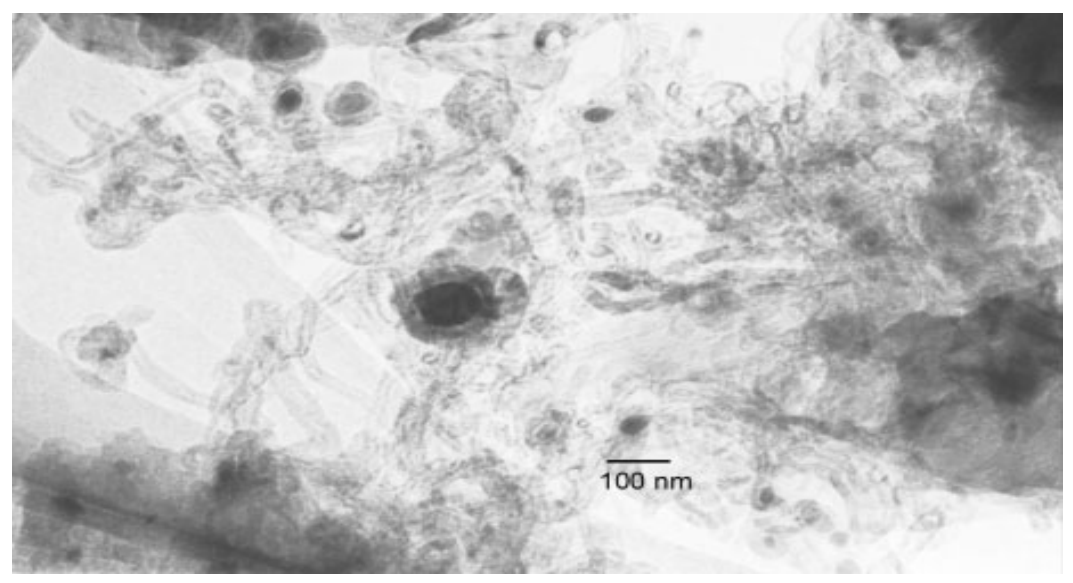

Fig. 4. TEM image showing carbon nanotube formation

the diffuse rings. These spots are due to crystalline Fe phases seen in the XRD pattern of the C:Fe50 sample. The approximate size of the iron nanoparticles varies from $10 \mathrm{~nm}$ to $100 \mathrm{~nm}$.

\subsection{Carbon nanotube formation}

In order to investigate the formation of nanotubes using the organic precursor, maleic anhydride, slight changes were made in the preparation procedure. The ferrocene, which acts as a catalyst in the formation of nanotubes was kept in an alumina boat in the higher temperature zone of the furnace inside the $70 \mathrm{~cm}$ long quartz tube. Maleic anhydride vapourizes from the lower temperature zone, flows through the decomposed ferrocene $\left(\sim 400^{\circ} \mathrm{C}\right)$ and the iron atom formed by the decomposition combines with the incoming hydrocarbon vapour, forming an intermediate compound of $\mathrm{Fe}_{3} \mathrm{C}$ and carbon nanotubes. Just one hour at the set temperature $\left(900^{\circ} \mathrm{C}\right)$ is sufficient for a good yield of powder. The carbon coating formed over the iron nanoparticles is initially spherical in shape. After reaching its elastic limit, these carbon layers results in formation of hollow cylindrical tubes [20]. Figure 4 shows a TEM image of the carbon nanotubes formed using our precursor.

\subsection{Magnetic Properties}

Figure 5 and 6 show the magnetization hysteresis loop of the as-prepared powder samples $\mathrm{C}: \mathrm{Fe} 05$ and $\mathrm{C}: \mathrm{Fe} 50$ at temperatures $300 \mathrm{~K}$ and $50 \mathrm{~K}$. We see that saturation magnetization $\left(\mathrm{M}_{S}\right)$ and coercivity $\left(\mathrm{H}_{C}\right)$ determined from the hysteresis loops depend on the iron content in the precursor. The saturation magnetization $\left(\mathrm{M}_{S}\right)$, coercivity $\left(\mathrm{H}_{C}\right)$, remanent magnetization $\left(\mathrm{M}_{R}\right)$, satura- 


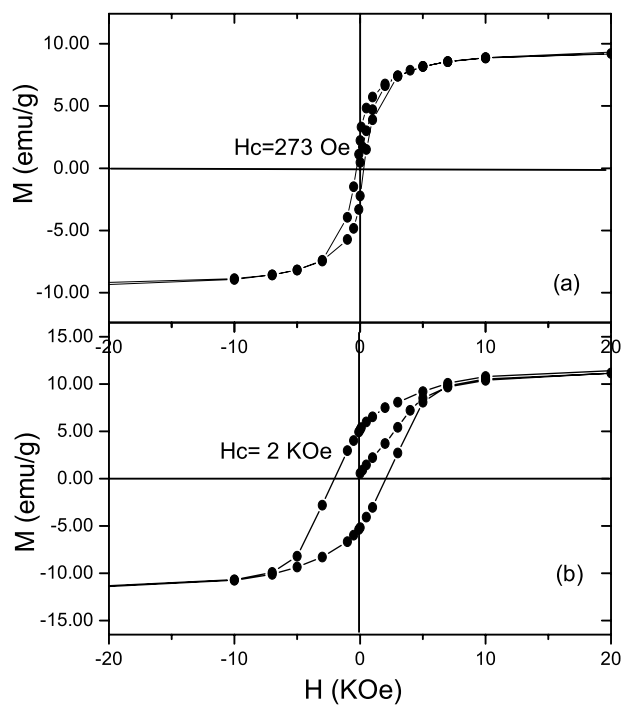

Fig. 5. Magnetization curve for C:Fe05 sample (a) 300K (b) 50K

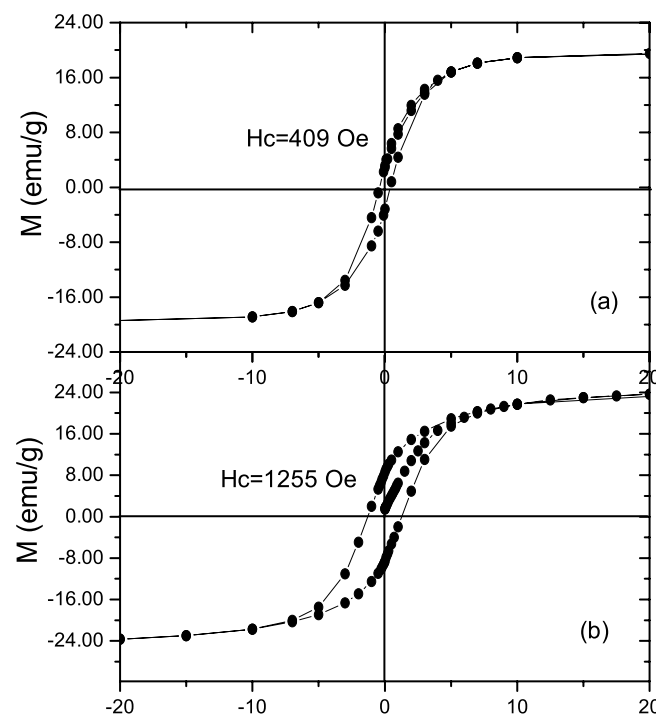

Fig. 6. Magnetization curve for C:Fe50 sample (a) 300K (b) 50K

tion magnetization per iron weight $\%\left(\mathrm{M}_{S}^{F e}\right)$ and the squareness ratio $(\mathrm{S})$ for both the samples are given in Table 2 .

The saturation magnetization $\left(\mathrm{M}_{S}\right)$ value of both samples increases with a decrease in temperature. If comparison is made taking into consideration the iron content present in the samples, the values approximately equal that of bulk iron carbide $(\sim 140 \mathrm{emu} / \mathrm{g})[9,18]$ at $50 \mathrm{~K}$. Even though $\alpha$-Fe possesses higher saturation magnetization $(212 \mathrm{emu} / \mathrm{g})$ than $\mathrm{Fe}_{3} \mathrm{C}$, its contribution to the $\mathrm{M}_{S}$ value is almost negligible. The reason for this is oxidation occuring around the $\alpha$-Fe ultrafine particles [9] thereby reducing the saturation magnetization. The presence of iron oxide in the C:Fe50 sample is confirmed by the 
Table 2

$\mathrm{M}_{S}, \mathrm{H}_{C}, \mathrm{M}_{R}$ and $\mathrm{M}_{R} / \mathrm{M}_{S}$ for C:Fe samples

\begin{tabular}{|c|c|c|c|c|c|c|}
\hline Temp. & Sample & $\begin{array}{c}\mathrm{M}_{S} \\
(\mathrm{emu} / \mathrm{g})\end{array}$ & $\begin{array}{c}\mathrm{H}_{C} \\
(\mathrm{Oe})\end{array}$ & $\begin{array}{c}\mathrm{M}_{R} \\
(\mathrm{emu} / \mathrm{g})\end{array}$ & $\begin{array}{c}\mathrm{M}_{S}^{F e} \\
(\mathrm{emu} / \mathrm{g} \text { of Fe })\end{array}$ & $\mathrm{S}=\mathrm{M}_{R} / \mathrm{M}_{S}$ \\
\hline \multirow{2}{*}{ 300K } & $\mathrm{C}: \mathrm{Fe} 05$ & 9.938 & 270 & 2.227 & 110.4 & 0.22 \\
& $\mathrm{C}: \mathrm{Fe} 50$ & 20.16 & 388 & 2.889 & 113.4 & 0.14 \\
& $\mathrm{C}: \mathrm{Fe} 05$ & 12.12 & 2000 & 5.15 & 134.7 & 0.42 \\
& $\mathrm{C}: \mathrm{Fe} 50$ & 24.44 & 1255 & 8.603 & 137.5 & 0.35 \\
\hline
\end{tabular}

XRD pattern. The ferromagnetic nature of the nanoparticles is characterized by the ratio of remanence to saturation magnetization $\left(\mathrm{M}_{R} / \mathrm{M}_{S}\right)$. The low remanence value of less than $25 \%$ clearly indicates that some of the particles are in a superparamagnetic state at room temperature. The contribution to the magnetization curve of the carbon matrix present in the $\mathrm{C}$ :Fe compositions is negligible. The amorphous carbon prepared from the organic precursor maleic anhydride alone is diamagnetic and the value of the magnetic moment is very small $(\mathrm{M}=0.03 \mathrm{emu} / \mathrm{g}$ for $\mathrm{H}=5.5$ tesla $)$ at room temperature. Hence, the background ferromagnetism due to the carbon matrix in our samples is completely eliminated.

The room temperature coercivity value for the C:Fe samples can be understood on the basis of the nucleation model of magnetic domain walls. Kittel [21] formulated particle size (diameter d) dependence of coercivity as,

$$
H_{C}=H_{C}^{\infty}\left(1-\frac{d}{d_{0}}\right)
$$

Here $H_{C}^{\infty}=2 \gamma / \delta M_{S}$ and $d_{o}=24 \gamma / M_{S}^{2}$, where $\gamma$ and $\delta$ denote the surface energy density of a Bloch wall and the wall thickness respectively. The coercive force depends on factors like magnetocrystalline anisotropy energy, particle shape, and surface effect. A large coercivity value for a larger particle size is observed and studied in a single domain magnetic particle system. Coercivity $\left(\mathrm{H}_{C}\right)$, increases with decreasing temperature, as expected for small particle systems, due to the pinning of domain walls in the particles $[21,22]$. In the case of our sample it is seen that the coercivity of $\mathrm{C}: \mathrm{Fe} 05$ increases to $2 \mathrm{kOe}$, higher than $\mathrm{C}: \mathrm{Fe} 50$ sample $\left(\mathrm{H}_{C}=1.5 \mathrm{kOe}\right)$. Chen et al. [4] reported that surface anisotropy dominates the magnetic properties of fine particle systems. They showed that in an assembly of spherical, random and noninteracting single-domain particles (diameter $d$ ) with surface anisotropy constant $K_{s}$, the coercivity is given by

$$
H_{C}=\frac{5.78 K_{s}}{M_{s} d}\left[1-\left(\frac{25 k T}{K_{s} \pi d^{2}}\right)^{1.155}\right]
$$


where $k$ and $T$ are the Boltzmann constant and the temperature respectively. When $\mathrm{d}$ is larger than $\mathrm{d}_{p}=\left(25 k T / \pi K_{s}\right)^{1 / 2}$; the upper particle limit for the superparamagnetic behaviour, the last term in the above equation can be neglected and the coercivity is thus inversely proportional to $d$, i.e., $H_{C} \propto 1 / d$. The presence of smaller particles in the C:Fe05 sample as seen in the TEM may be a possible explanation to the increase in coercivity. Xiao et. al. [2] have reported a giant coercivity effect in Fe granular films; about 600 Oe at room temperature as well as 2500 Oe at $2 \mathrm{~K}$; showing sensitivity to grain size. There have been various reports [3] on the magnetic properties of fine particles, where coercivity $\mathrm{H}_{C}$ decreases according to the $1 / d$ relationship. The coercivity mechanism in our C:Fe system is thus a complex mixture of several mechanisms, including nucleation, pinning of domain walls, superparamag-

netism and coherent rotation. Work is still underway to study the effect of graphitization and pyrolysis temperature on the magnetic properties of these materials.

\section{Conclusions}

A chemical vapour deposition technique has been used to prepare C:Fe samples. The samples consisted of iron nanoparticles embedded in a carbon matrix. For lower iron concentrations the overwhelming majority of the $\mathrm{Fe}_{3} \mathrm{C}$ nanocrystallites are embedded in a carbon matrix. The basic ferromagnetic character of the sample was given by the $\mathrm{M}_{R}$ to $\mathrm{M}_{S}$ ratio and the value of saturation magnetization per gram for the two samples. The high coercive force of the particles even at room temperature shows that the material can be used in practical applications.

\section{Acknowledgements}

E.P Sajitha thanks the Central facility, Department of Physics, IISc for carrying out XRD measurements on the samples and the late Prof.G.N Subbana for his help in carrying out TEM observations.

\section{References}

[1] McHenry ME, Majetich SA, Artman JO, DeGraef M, Staley SW. Superparamagnetiam in carbon-coated Co particles produced by the Kratschmer carbon arc process. Phys. Rev. B 1994;49:11358-63

[2] Xiao G, Chien CL. Giant magnetic coercivity and percolation effects in granular Fe- $\left(\mathrm{SiO}_{2}\right)$ solids. Appl. Phys. Lett. 1987;51:1280-82 
[3] Liou SH, Chien CL. Granular metal films as recording media. Appl. Phys. Lett. $1988 ; 52: 512-14$

[4] Chen C, Hitakami O, Okamoto S, Shimada Y. Surface anisotropy in giant magnetic coercivity effect of cubic granular $\mathrm{FeCo} / \mathrm{SiO}_{2}$ and $\mathrm{NiCo} / \mathrm{SiO}_{2}$ films: A comparison with Neel's Theory. J. Appl. Phys. 1999;86(4):2161-65

[5] Wang ZH, Zhang ZD, Choi CJ, Kim BK. Structure and magnetic properties of $\mathrm{Fe}(\mathrm{C})$ and $\mathrm{Co}(\mathrm{C})$ nanocapsules prepared by chemical vapor condensation. J. Alloys Comp. 2003;361:289-93

[6] Hihara T, Onodera H, Sumiyama K, Suzuki K, Kasuya A, Nishina Y, et al. Magnetic properties of iron in nanocapsules. Jpn. J. Appl. Phys. 1994;33:L2425

[7] Si PZ, Zhang Z, Geng D, You C, Zhao X, Zhang W. Synthesis and characteristics of carbon-coated iron and nickel nanocapsules by arc discharge in ethanol vapour. Carbon 2003;41:247-51

[8] Liu Y, Ling J, Li W, Zhang X. Effective synthesis of carbon-coated Co and Ni nanocrystallites with improved magnetic properties by AC arc discharge under an $\mathrm{N}_{2}$ atmosphere. Nanotechnology 2004;15:43-47

[9] Zhao XQ, Liang Y, Hu ZQ, Liu BX. Oxidation characteristics and magnetic properties of iron carbide and iron ultrafine particles. J. Appl. Phys. 1996;80:5857-60

[10] Masuda M, Maeda K, Kobayashi T, Shiomi S, Fujiwara Y, Saito Y. Synthesis, crystal structure and magnetic properties of iron particles encaged in carbon nanocapsules Jpn. J. Appl. Phys. 2000;39:L733-34

[11] Scott JHJ, Majetich SA. Morphology, structure, and growth of nanoparticles produced in a carbon arc Phys. Rev. B 1995;52:12564-571

[12] Tartaj P, Morales MP, Veintemillas-Verdaguer S, Gonzalez-Carreno T, Serna CJ.The preparation of magnetic nanoparticles for applications in biomedicine.J. Phys. D: Appl. Phys.2003;36:R182-97

[13] Hayashi T, Hirono S, Tomita M, Umemura S, Magnetic thin films of cobalt nanocrystals encapsulated in graphite-like carbon. Nature 1996;381:772-74

[14] Delaunay JJ, Hayashi T, Tomita M, Hirono S, Umemura S,CoPtC nanogranular magnetic thin films. Appl. Phys. Lett. 1997; 71:3427-29

[15] Song H, Chen X. Large scale synthesis of carbon-encapsulated iron carbide nanoparticles by co-carbonization of durene with ferrocene. Chem. Phys. Lett. 2003;374:400-04

[16] Sano N, Akazawa H, Kikuchi T, Kanki T. Separated synthesis of iron-included carbon nanocapsules and nanotubes by pyrolysis of ferrocene in pure hydrogen. Carbon 2003;41:2159-79 
[17] Xiang-Xin Bi, Ganguly B, Huffman GP, Huggins FE, Endo M, Eklund PC. Nanocrystalline $\alpha$-Fe, $\mathrm{Fe}_{3} \mathrm{C}$, and $\mathrm{Fe}_{7} \mathrm{C}_{3}$ produced by $\mathrm{CO}_{2}$ laser pyrolysis. J. Mater. Res. 1993;8:1666-74

[18] Liu X, Huang B, Coville NJ. The $\mathrm{Fe}(\mathrm{CO})_{5}$ catalyzed pyrolysis of pentene: carbon nanotube and carbon nanoball formation. Carbon 2002;40:2791-99

[19] Pan L, Zhang M, Nakayama Y Growth mechanism of carbon nanocoils. J. Appl. Phys. 2002;91:10058-61

[20] Yudasaka M, Kikuchi R. Graphitization of Carbonaceous Materials by Ni, Co and Fe. Yoshimura S, Chang RPH, Editor. Supercarbon,Springer,1998:99-105

[21] Kittel C. Physical theory of ferromagnetic domains. Rev. Mod. Phys.1949;21:541-83

[22] Zhang XX, Wen GH, Huang S, Dai L, Gao R, Wang ZL. Magnetic properties of Fe nanoparticles trapped at the tips of the aligned carbon nanotubes. J. Magn. Magn. Mater. 2001;231:L9-12 